\title{
Learning Styles Adaptation Language for Adaptive Hypermedia
}

\author{
Natalia Stash, Alexandra Cristea, Paul De Bra \\ Faculty of Mathematics and Computer Science, Eindhoven University of Technology, \\ Postbus 513, 5600 MB Eindhoven, The Netherlands \\ \{nstash, acristea, debra\}@win.tue.nl
}

\begin{abstract}
Typically, the behavior of adaptive systems is specified by a set of rules that are hidden somewhere in the system's implementation. These rules deal with instances of the domain model. Our purpose is to specify the adaptive response of the system at a higher level (to be applied and reused for different domains or adaptive applications) in an explicit form, called adaptation language. For this purpose we have chosen learning styles (LS) as an implementation field. We defined an XML-based adaptation language LAG-XLS for the AHA! system. In this paper we focus on the empirical evaluation of LAG-XLS.
\end{abstract}

\section{Introduction}

Adaptive hypermedia (AH) systems (AHS) mostly focus on the delivery of adaptive applications to end-users and less on authoring aspects [2]. To allow a widespread use of AHS, more attention is needed for the authoring process [2], to make it as "simple" and intuitive as possible [5]. In [9], to alleviate the so-called "authoring problem" we discussed limiting repetitive work by reuse of previously created materials and other components. These include the static parts of the authored courseware (e.g., domain model content) and the actual system dynamics (adaptive behavior). Most existing standards (LOM, SCORM, etc.) address only static and not dynamic reuse [9]. In [9] we compared LAG-XLS ('LAG-excels'), a language developed for the AHA! (Adaptive Hypermedia Architecture) system [7], with a more generic language for AH, LAG $[3,5]$, as its theoretical basis. LAG-XLS focuses on adaptation to various learning styles (LS), meaning here an individual's preferred way of learning. Here we outline what type of strategies can be created in LAG-XLS, how they are applied and visualized in AHA! applications, and we present some evaluation results of our approach.

\section{Adaptation to Learning Styles in AHA!}

LAG-XLS: allows 3 types of adaptive behavior [9]: selection of items to present (e.g. media types); ordering information types (e.g., examples, theory, explanation); and creating different navigation paths (e.g. breadth-first vs. depth-first). LAG-XLS also 
allows for the creation of meta-strategies, tracing users' preferences for certain types of information or reading order. Strategies are defined as XML files using a predefined DTD. XML was chosen as it is an extensible language and a W3C standard.

Creating an AHA! adaptive application: consists of defining the domain/adaptation model and writing application content (XHTML pages [7]). The extended system allows applying adaptive strategies, specified in LAG-XLS, to the domain model. Authors can create their own strategies or reuse existing ones. We pre-defined adaptation strategies for the following LS [8,3]: active vs. reflective, verbalizer vs. imager (visualizer), holist (global) vs. analytic, field-dependent vs. field-independent; strategies for inferring user preferences (adaptation meta-strategies) for textual or pictorial information, and navigation in breadth-first or depth-first order (BF vs. DF). Authors can change the predefined strategies. For this, they have to use elements defined in the LAG-XLS DTD, and ensure that the domain model concepts have the attributes required by the strategies [9]. Authors choose which strategies to apply to a particular application, and in which order (in case of several strategies, order can be important).

Visualization of strategies application in AHA!. The learner sets his preferences (e.g., LS) via a form or selects preference tracing. Later a user can inspect his user model and make changes to it (e.g., to try a new strategy corresponding to other LS).

\section{Empirical Evaluation LAG-XLS}

\subsection{Evaluation Settings}

We tested the application of LAG-XLS (meta-)instructional adaptation strategies to AHA! in an AH course [1], with 34 students: $4^{\text {th }}$ year undergraduates in Computer Science and $1^{\text {st }}$ year Masters students in Business Information Systems.

\subsection{The Experimental LAG-XLS Assignment Steps}

1. The students had to perform the assignment in groups of 2-3 people in 4 weeks. 2. They installed the AHA! system version supporting LS on their notebooks. It contained two example applications (courses) and some predefined strategies to apply. 3. Students had 2 roles: authors; using the Graph Author tool [7] to see course concept structure and select strategies to apply; end users: visualizing strategy application results; analyzing the same course with different LS settings and automatic tracing. 4. Next, the students filled out a questionnaire about their experience with the system. 5. The students also filled out the Felder-Solomon "Index of Learning Styles Questionnaire" (ILS) [8]. ILS maps a set of 44 questions over 4 LS dimensions. For the assignment, 3 dimensions were of interest: active vs. reflective, visual vs. verbal and sequential vs. global. We examined if the students' preferred settings (explicitly selected by them whilst using the system) corresponded to the LS revealed by the ILS questionnaire and if the system's inferred preferences matched the ILS questionnaire. 6 . Finally, students were asked to create their own strategies, or variations of existing strategies, in the LAG-XLS language, and apply them in the provided applications. 


\subsection{Experimental quantitative results}

The quantitative results of the assignment are presented in the integrated table 1 .

Table 1. Experimental quantitative results

\begin{tabular}{|c|c|c|c|c|c|c|}
\hline \multirow{2}{*}{$\begin{array}{l}\text { 1. Students' average } \\
\text { stated preferences }(\%)\end{array}$} & verbal & visual & active & reflective & global & analytic \\
\hline & 9 & 68 & 24 & 56 & 24 & 41 \\
\hline \multirow{2}{*}{$\begin{array}{l}\text { 2. ILS questionnaire } \\
\text { average results (\%) }\end{array}$} & verbal & visual & active & reflective & global & sequential \\
\hline & 1 & 49 & 19 & 9 & 26 & 5 \\
\hline \multirow{2}{*}{$\begin{array}{l}\text { 3. Students' prior knowl- } \\
\text { edge }(\%)\end{array}$} & \multicolumn{3}{|c|}{ pre-knowledge LS } & \multicolumn{3}{|c|}{ pre-knowledge XML } \\
\hline & \multicolumn{3}{|c|}{24} & \multicolumn{3}{|c|}{79} \\
\hline \multirow{2}{*}{$\begin{array}{l}\text { 4. Overall impression of } \\
\text { instructional strategies } \\
\text { and experiments }(\%)\end{array}$} & useful & \multicolumn{3}{|c|}{ pleasant } & \multicolumn{2}{|c|}{ easy } \\
\hline & 82 & \multicolumn{3}{|c|}{67} & \multicolumn{2}{|c|}{54} \\
\hline \multirow[t]{2}{*}{$\begin{array}{l}\text { 5. Working with the } \\
\text { system }(\%)\end{array}$} & $\begin{array}{l}\text { understan } \\
\text { Graph } \\
\text { Author }\end{array}$ & \multicolumn{2}{|c|}{$\begin{array}{l}\text { no prob- } \\
\text { lem } \\
\text { editing? }\end{array}$} & $\begin{array}{l}\text { understand } \\
\text { application } \\
\text { strategies }\end{array}$ & $\begin{array}{l}\text { satisfied } \\
\text { with } \\
\text { presenta- } \\
\text { tion }\end{array}$ & $\begin{array}{l}\text { strategy } \\
\text { change } \\
\text { worked? }\end{array}$ \\
\hline & 88 & \multicolumn{2}{|c|}{47} & 77 & 76 & 75 \\
\hline \multirow[t]{2}{*}{$\begin{array}{l}\text { 6. Students' satisfaction } \\
\text { with the strategies }(\%)\end{array}$} & $\begin{array}{l}\text { verbalizer } \\
\text { vs. image }\end{array}$ & \multicolumn{2}{|c|}{$\begin{array}{l}\text { active vs. } \\
\text { reflective }\end{array}$} & $\begin{array}{l}\text { global vs. } \\
\text { analytic }\end{array}$ & $\begin{array}{l}\text { text vs. } \\
\text { image } \\
\text { pref. }\end{array}$ & $\begin{array}{l}\text { BF vs. DF } \\
\text { pref. }\end{array}$ \\
\hline & 87 & \multicolumn{2}{|c|}{67} & 73 & 87 & 71 \\
\hline
\end{tabular}

Students' stated preferences vs. ILS questionnaire results. Table 1 (row 1, 2) shows that students are rarely aware of their LS. Note the difference between stated "analytic" (equivalent here with "sequential") preference and the ILS results (showing "global" tendency). Further on, for "active vs. reflector", the former tendency is stronger in ILS, whilst the latter dominates in actual use. Results coincide in the students' strong image preference. Still, the intensity is different in praxis and theory.

Students' prior knowledge (row 3). As most were from computer science, unsurprisingly, their XML prior knowledge was far greater than the LS one (79 vs. 24\%). Many had never heard of LS before. This may explain fluctuations in learning preferences.

Students' general impression of their first encounter of $L S$ in combination with $A H$ (row 4). Students considered the implementation of adaptive instructional strategies and (monitoring) meta-strategies for adaptive educational systems useful (82\%). Less strong, but still positive was their conviction about this experimental process being pleasant $(67 \%)$. A (smaller) majority of students considered the work easy $(54 \%)$. This difference shows that, although students realized the necessity and importance of adaptive strategies in $\mathrm{AH}$, and enjoyed the assignment, they did not consider it trivial. Thus reuse of ready-made, custom-designed strategies is vital for $\mathrm{AH}$ authors, to reduce creation time and costs.

Working with the system (row 5). The students understood how the application of strategies works $(77 \%)$ - the core of the LAG-XLS language understanding - and are greatly satisfied with the presentations (76\%). They understand the AHA! Graph Author very well $(88 \%)$. However creation of their own strategies was the most difficult problem. Only $47 \%$ did not have a problem with editing. The strategy changes worked well for $75 \%$ of the students. 
Students' satisfaction with the (meta-)strategies (row 6). All strategies and metastrategies were deemed appropriate by the majority (over 65\%) of students. The "winning" strategy is the "verbalizer vs. imager", considered most accurate (87\%). Following is the "global vs. analytic" (73\%) and "activist vs. reflector" strategy (67\%). From the meta-strategies, the one liked best by students was the "text vs. image preference" meta-strategy. For the latter, most students noticed that it traced their behavior within 3 steps. The "BF vs. DF" strategy is more complex. For a user with a breadth-first preference, the system analyzes a larger number (between 7-14, with an average:13) of steps till the LS was detected. $71 \%$ were satisfied with the strategy.

\subsection{Experimental qualitative results: selection of Questions and Answers}

Due to the of lack of space, we only provide a summary of some of the comments.

1. Do you find the application of different instructional/monitoring strategies for educational adaptive hypermedia useful? Most students gave a positive reply. They considered it a good aid in the learning process, as presentation of material suiting the user's need allows working more efficiently and saves time. However, some correctly noticed that it is quite easy to fool the system, as it does not check whether material is really understood when the user browses through it (this is a typical AH problem).

2. Compare the preference induced by the system with the ILS questionnaire results. For the majority of students the induced preference corresponded with the ILS results. If this was not the case the students provided us with some comments. One student replied: "I generally like to see the global picture first and then go into the details. However in the tutorial, ... If I read the high level concepts first and then go into the details, I have forgotten what the high level concepts were." This problem may be caused by the fact that the authors of the example application do not have enough psychological knowledge about how to fully support the global and analytic LS (beyond the recommended breadth-first and depth-first processing). Another student commented that he has a textual preference according to ILS, but he so much liked the pictures in the tutorial that he preferred the imager version. Also, some students correctly noticed that LS preferences may vary in different domains.

3. Can you think of more strategies that you would like to apply but are not able to express using $\boldsymbol{L A G}$-XLS? Most students were only able to create variations of the existing strategies by using different names for presentation items and by increasing/decreasing the number of steps required by the monitoring strategies to achieve a threshold. The students did not come up with any completely new strategies.

\section{Discussion and Conclusion}

From the evaluation results we can say that designing an application in such a way that different types of users get equivalent information appropriate to them is a useful endeavour. Students understood the process and liked being involved in it, in spite of the fact that it wasn't a simple task. It is very reassuring that our students understood the basics of LS application, as they were computer science students, with little or no 
knowledge in this field prior to the course. This exercise shows also the challenges of the end-user side, the learner: theory and praxis do not always match in identification of LS. The end-user rarely has meta-knowledge of this type.

This was a small-scale exercise in authoring the dynamics of $A H$, from the point of view of tasks involved (the group size was average). The results and comments show that LAG-XLS allows a quick grasp on the adaptation process (for computer science students), as well as relatively easy handling and small modifications of existing adaptation strategies. Still, some students couldn't create new strategies from scratch.

It is clear that the creation process of adaptive behaviour in itself requires a lot of psychological and/or pedagogical knowledge. As we are no psychologists, the main aim of our research is to allow the authors with experience in pedagogical psychology to design different types of strategies and apply these strategies to the applications. Moreover, the question about how to structure the application and organization of the materials to correctly suit different LS is left for the author of the application or psychologist. Therefore, from a future evaluation point of view, it would be interesting to test LAG-XLS with LS specialists, instead of computer scientists, focusing more on the qualitative aspects instead of the technical aspects of the language.

\section{Acknowledgements}

This work is supported by the PROLEARN network of excellence, the NLnet Foundation and was initiated by the EU Minerva ADAPT project.

\section{References}

1. Adaptive Hypermedia course (TU/e, 2ID20) winter trimester 2004/05, http://wwwis.win.tue.nl/ acristea/AH/

2. Brusilovsky, P., Developing adaptive educational hypermedia systems: from design models to authoring tools. "Authoring Tools for Advanced Technology Learning Environments", Eds. T. Murray, S. Blessing, S. Ainsworth, Kluwer (2003).

3. Coffield, F., Learning Styles and Pedagogy in post-16 learning: A systematic and critical review. Learning \& Skills research centre. http://www.lsda.org.uk/files/pdf/1543.pdf

4. Cristea, A.I., and Calvi, L. The three Layers of Adaptation Granularity. UM'03. Springer.

5. Cristea, A.I., and Verschoor, M. The LAG Grammar for Authoring the Adaptive Web, ITCC'04 April, 2004, Las Vegas, US, IEEE (2004).

6. Cristea, A. Authoring of Adaptive Hypermedia; Adaptive Hypermedia and Learning Environments; "Advances in Web-based Education: Personalized Learning Environments". Eds.: S. Y. Chen \& Dr. G. D. Magoulas. IDEA Publishing group (2006).

7. De Bra, P., Stash, N., Smits, D., Creating Adaptive Web-Based Applications, Tutorial at the 10th International Conference on User Modeling, Edinburgh, Scotland (2005)

8. R. M. Felder \& B. A. Soloman 2000. Learning styles and strategies. At URL: http://www.engr.ncsu.edu/learningstyles/ilsweb.html

9. Stash, N., Cristea, A., De Bra, P. Explicit Intelligence in Adaptive Hypermedia: Generic Adaptation Languages for Learning Preferences and Styles, HT'05, CIAH Workshop, Salzburg, (2005) 